\title{
Advances in the detection of phycotoxins and cyanotoxins
}

\section{J. F. Humbert}

Published online: 23 May 2010

(C) Springer-Verlag 2010

Photosynthetic microorganisms play an important role in the functioning of aquatic ecosystems, because they are involved in much of the primary production, and form the basis of trophic networks. However, these microorganisms can also be harmful, because many species are able to produce toxins that threaten both animal and human health. These organisms and their toxins can disrupt aquatic ecosystems. In freshwater ecosystems this disruption mainly affects the production of drinking water and the use of water for recreational activities, in particular for bathing. In marine environments, the main risk concerns human consumption of contaminated organisms (notably shellfish, etc.). The numerous poisoning events that have resulted from the consumption of contaminated water or food throughout the world have led to monitoring programs to survey potentially toxic microorganisms and their toxins. All these programs involve a wide range of tools, including microscopic examination, and analytical or bioanalytical methods. Numerous research programs are also in progress that are intended to improve these tools or to provide new methods, with the aim of reducing the cost and the time required to carry out these analyses, and also to reduce the health risks for human populations. The articles in this special issue of Analytical and Bioanalytical Chemistry illustrate some of these recent developments, and try to identify the strategies that are likely to be used in the future to survey photosynthetic microorganisms and their toxins in aquatic ecosystems.

J. F. Humbert ( $\square)$

INRA, UMR BIOEMCO,

Site de l'ENS, 46 rue d'Ulm,

75005 Paris, France

e-mail: humbert@biologie.ens.fr
This issue includes three papers dealing with surveys of marine toxins, in particular those found in seafood. This is a high-priority topic owing to the impact of these toxins on human health, and also because of their potential financial repercussions. The recent uproar in France when the sale of shellfish was banned provides a good illustration of the need for rapid and reliable methods of risk assessment. The first paper, by Humpage et al., focuses on paralytic shellfish poisoning toxins, which have been involved in numerous human poisoning events worldwide. Moreover, the recent discovery that these toxins can also be produced by freshwater cyanobacterial species, and consequently that drinking water is another potential route of human exposure, has stimulated numerous methodological developments intended to provide alternatives to the mouse bioassay. Two other papers also concern marine toxins that can contaminate seafood. Vilariño et al. propose an overview of the alternatives to animal bioassays, and focus particularly on biosensors and functional methods. The paper by Hess compares the effectiveness of various different detection methods used in Irish and French monitoring programs, and makes suggestions in the area of phycotoxin detection based on recent expert assessments.

Potentially toxic marine species and their toxins have been monitored for more than 20 years in many countries, owing to the early awareness of the health risks associated with these toxins, but surveys of cyanobacteria and of their toxins in freshwater ecosystems are a much more recent development. In France, for example, the French Marine Phytoplankton Monitoring Network (REPHY) was set up by IFREMER in 1984, whereas monitoring of cyanobacteria and cyanotoxins in freshwater ecosystems only began in the 2000s. For this reason, the detection of cyanobacteria and of their toxins has been the topic of numerous studies in the last 10 years. We need new tools to provide the rapid 
and accurate detection of toxins in the field required for various avenues of research in this area. The paper by Khreich et al. describes a new and very sensitive immunochromatographic test based on the use of immunoliposomes to detect microcystins and nodularins. This test will make it possible to use a hand-held fluorometer to quantify these toxins in the field. Another topic of current interest in the field of cyanotoxin detection concerns the detection of neurotoxins, particularly that of anatoxins. The paper by Araoz et al. provides a good overview of the methods available, with a particular focus on ligand-binding assays, which can also be used for the high-throughput screening of unknown nicotinic agents. The detection of cyanotoxins in water is crucial, but ways to remove or inactivate these toxins in drinking water are also a very important topic. The review by Westrick et al. provides a good overview of progress in this area, particularly with regard to water treatment processes that can remove the most frequently occurring cyanotoxins from potable water, and also how multibarrier approaches can contribute to providing safer water supplies for human populations.

Finally, the last two papers in this special issue concern both phycotoxins and cyanotoxins. The first of these papers, by Fessard and Le Hegarat, addresses the very important question of evaluating the genotoxicity of these toxic compounds. This paper highlights the fact that the same strategies can be used to evaluate both marine and freshwater toxins, and also that one of the most critical limitations of these evaluations results from the lack of standards for many of these toxins. This point was also mentioned in several of the papers dealing with toxin detection, demonstrating the urgent need for standards for these toxic substances, whether for the evaluation of the toxic risks they pose or to detect them in the environment.

These analytical and bioanalytical approaches will doubtless remain the reference survey methods for phycotoxins and cyanotoxins in the coming years, but the tools offered by molecular biology are just beginning to provide alternative ways to survey marine and freshwater microorganisms and their toxins in aquatic ecosystems. The paper by Humbert et al. shows that many tools have been developed in recent years, and that some of them are already fully operational in the framework of monitoring programs. From this review, it appears that the great advantage of these approaches will be to permit the early detection of toxic microorganisms in water, and thus to anticipate problems that could result from their development in aquatic ecosystems.

As guest editor, I am very pleased with the papers included in this special issue of Analytical and Bioanalytical Chemistry, because I feel they are truly representative of the diversity and quality of the research being performed in the fields of phycotoxin and cyanotoxin detection and of risk assessment. I would like to conclude this editorial by thanking all the authors for their excellent contributions to this issue, and the editorial team of Analytical and Bioanalytical Chemistry for their fruitful assistance.

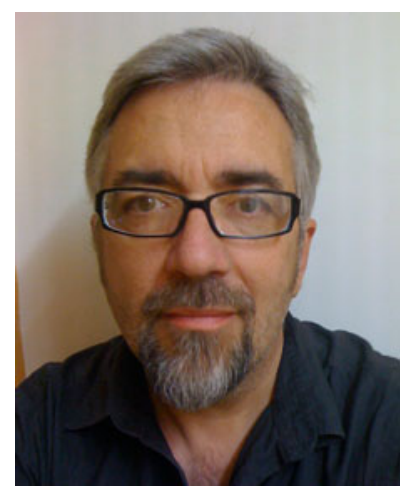

J.F. Humbert is currently a senior researcher (directeur de recherche) at the Institut National de la Recherche Agronomique (INRA, France), and he works at UMR Bioemco in Paris. He is involved in both basic and applied research work on the microbial communities of freshwater ecosystems, and has a special interest in toxic cyanobacteria. In recent years he has been involved in several programs investigating the genetic and genomic diversity of these microorganisms, and also in developing new tools for monitoring cyanobacteria and their toxins in freshwater ecosystems. 